\title{
Biomechanical evaluation of central and peripheral Descemet's membrane endothelial graft
}

Vito Romano ${ }^{1,2}$, Zhuola ${ }^{1}$, Zhuo Chang ${ }^{3}$, Bernhard Steger ${ }^{4}$, Hannah J. Levis ${ }^{2}$, Stephen B. Kaye ${ }^{1,2}$, Riaz Akhtar ${ }^{3}$

${ }^{1}$ Department of Ophthalmology, St. Paul's Eye Unit, Royal Liverpool University Hospital, Liverpool, UK; ${ }^{2}$ Department of Eye and Vision Science, Institute of Ageing and Chronic Disease, University of Liverpool, Liverpool, UK; ${ }^{3}$ Department of Mechanical, Materials and Aerospace Engineering, School of Engineering, University of Liverpool, Liverpool, UK; ${ }^{4}$ Department of Ophthalmology, Medical University of Innsbruck, Innsbruck, Austria

\section{Abstract}

Corneal endothelial transplant is the gold-standard treatment in cases of corneal endothelial cellular dysfunction. Preparation, delivery, and unfolding of the graft are technically demanding. We assessed the biomechanical behavior of Descemet's membrane to better understand Descemet's membrane endothelial keratoplasty (DMEK) graft behavior, and to select the right diameter and donor age graft. The biomechanical behavior was tested using atomic force microscopy (AFM) on five corneas unsuitable for transplantation. The peripheral cornea was found to be stiffer than the central cornea (3171.89 MPa and 2837.20 MPa, respectively). The elastic modulus of both the central and peripheral cornea exhibited a trend to decrease with age. In addition, the central cornea becomes stiffer than the peripheral cornea in older patients, while the peripheral cornea was stiffer in younger patients. AFM is a suitable technique for evaluating biomechanical behavior of DMEK grafts. One interpretation of this varied behavior is that the type and quantity of collagen changes with age and location.

Keywords: atomic force microscopy (AFM), biomechanical behavior, corneal transplant, endothelial keratoplasty

Correspondence: Vito Romano, MD, Department of Corneal and External Eye Diseases, St Paul's Eye Unit, Royal Liverpool University Hospital, Prescot Street, Liverpool, L7 8XP, UK. E-mail:vito.romano@gmail.com 


\section{Introduction}

Over the last twenty years, significant advances have been made in corneal transplantation techniques. The treatment of corneal pathologies has evolved from the replacement of full-thickness cornea to replacing only the diseased corneal endothelium. ${ }^{1}$ During the past several years, new surgical techniques have been reported, endothelial keratoplasty being the gold standard. Posterior lamellar keratoplasty, either Descemet's stripping automated endothelial keratoplasty (DSAEK) or DMEK, eliminate surface corneal incisions or sutures, maintain much of the cornea's structural integrity, and induce minimal refractive change, suggesting distinct advantages over standard penetrating keratoplasty. DMEK represents the final iteration of endothelial keratoplasty (EK), producing near-pure anatomic replacement therapy for patients with endothelial dysfunction, stromal edema, and Descemet's membrane disfigurement. Although the visual results after DMEK are better on average than the published visual results after DSAEK, DMEK has not overtaken DSAEK because it is a technically demanding surgical procedure. ${ }^{2}$ Handling the DMEK graft is difficult, making loading, delivery, and unfolding very challenging, thus taking up surgical time..$^{3-7}$ In order to better understand DMEK graft behavior and to select the right diameter and donor age graft, we studied the biomechanical behavior of the Descemet's membrane graft.

\section{Purpose}

To characterize the biomechanical behavior of Descemet's membrane, its parts, and the DMEK graft.

\section{Design}

Laboratory study.

\section{Methods}

Five corneas unsuitable for transplantation were collected from the Manchester Eye Bank Foundation, UK, with a written consent to be used for research from the donor's next of kin. The donor corneas did not show indications of any systemic disease, dystrophy, or infections. The corneas were not suitable for transplant due to low endothelial cell count.

The average age of the donors was between 54 and 77 years. The tissues were preserved in organ culture medium (OCM) for 20 days. 
All the corneas $(n=8)$ were centered on the base of a trephine punch using the peripheral holes of the suction area as reference. A 9.5- $\mathrm{mm}$ diameter punch (Moria, SA: Antony, France) was used to create a superficial cut by gently tapping the endothelial surface (cut edge) of the tissue. The endothelium was submerged in the OCM. The membrane was lifted using a cleavage hook throughout the circumference to limit the peripheral tearing of the very fragile DMEK tissue. Using acute forceps (E. Janach SRL; Como, Italy), the membrane was stripped with a longitudinal movement from three sides, ensuring that no torsions were generated during this phase.

The nanomechanical properties were measured with AFM using PeakForce Quantitative Nanomechanical Mapping (QNM) in air mode. The AFM probe had a $5 \mathrm{~N} / \mathrm{m}$ of nominal spring constant of the cantilever and $8 \mathrm{~nm}$ of nominal tip radius. The resonant frequency was $150 \mathrm{kHz}$. For each sample, three random areas $\left(5 \mu \mathrm{m}^{2}\right)$ were imaged in the center and periphery of the corneas. The DMEK grafts were tested with the corneal endothelium facing downwards, to expose Descemet's membrane to the probe.

For the nanoindentation measurements, the Peak Force set point was fixed at $0.05 \mathrm{~V}$, which gives rise to a maximum force of about $5 \mathrm{nN}$. For the data processing and elastic modulus calculation, unloading curves were utilized. It is worth pointing out that negligible hysteresis was observed during the approaching-retraction cycles, reasonably because of the absence of cells on the non-endothelial side of the membrane.

Statistical analysis was performed using Stata (StataCorp; College Station, Texas, USA) and a Student's t-test was performed, deeming $\mathrm{p}<0.05$ statistically significant.

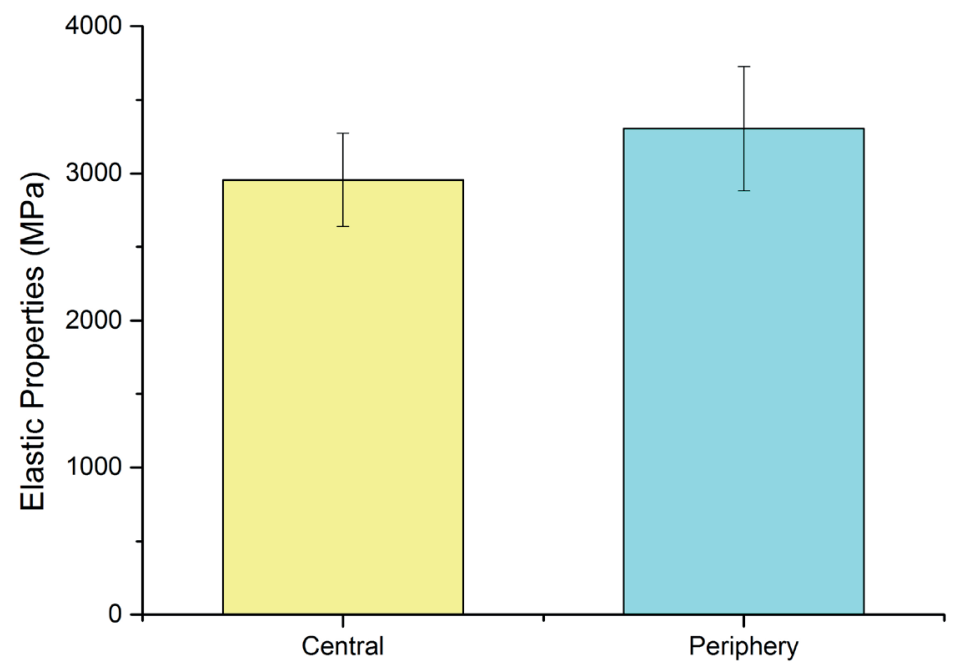

Fig. 1. Elastic properties in MPa of the central and peripheral cornea. 


\section{Central}

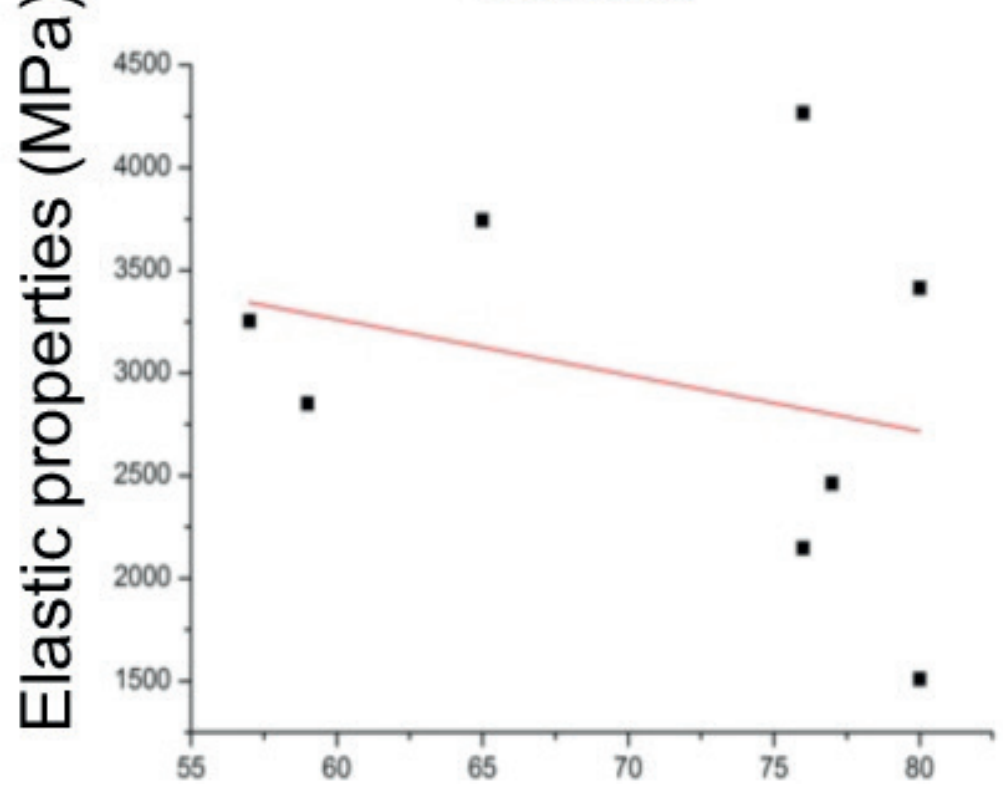

Fig. 2. Elastic modulus of the central cornea.

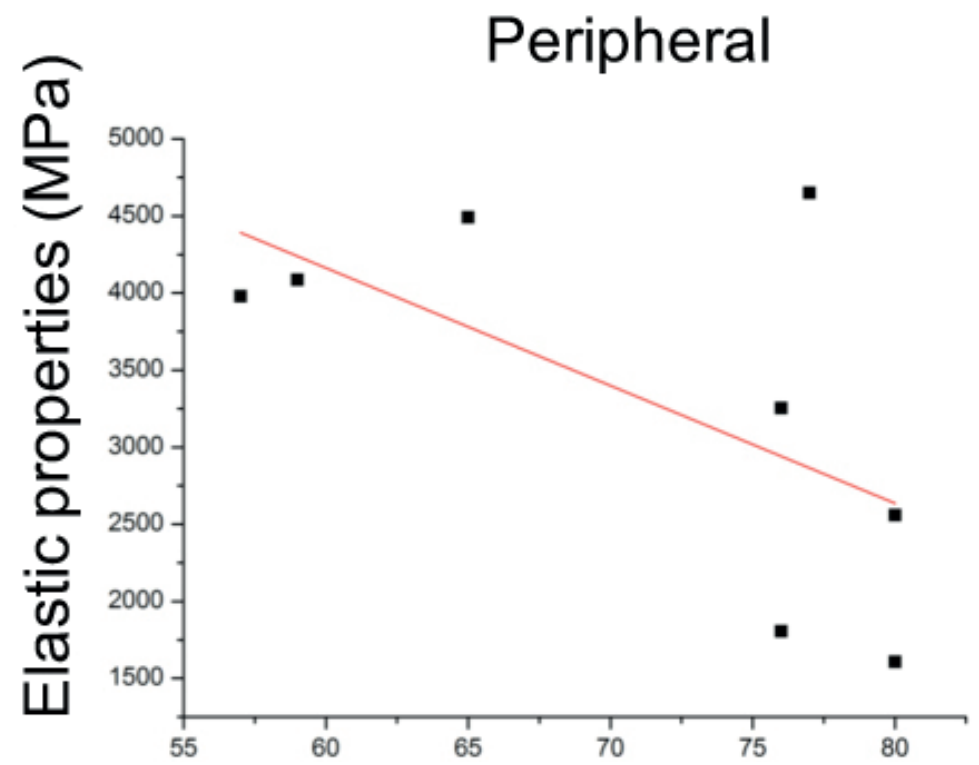

Fig. 3. Elastic modulus of the peripheral cornea. 


\section{Results}

The periphery of the cornea was stiffer than the center $\left(\mathrm{E}_{\text {periphery }}=3171.89 \mathrm{MPa}\right.$; $E_{\text {center }}=2837.20 \mathrm{MPa}$ ) (Fig. 1). The elastic modulus of both the central and peripheral cornea exhibited a trend to decrease with age. Interestingly, we found that the center was stiffer than the periphery in older patients (75-80 years) (Fig.2), while the periphery was stiffer in younger patients (55-70 years) (Fig.3).

\section{Conclusion}

Peakforce AFM is a suitable technique for evaluating DMEK grafts. The limitation of the present study is the limited number of samples considered, which reduces the statistical significance of the results, and thus, the lack of stratification on a larger sample that can include a variability in age. Nevertheless, the study clearly showed different behaviors between the central and peripheral DMEK graft, which can be explained in terms of a different distribution in collagen quantity and quality. These findings provide some interesting areas for consideration and future research on the characterization of the biomechanical properties of the cornea for identification of potential risk factors in donor selection for DMEK surgery, allowing a potential reduction in complication rates and improved graft survival.

\section{References}

1. Anshu A, Price MO, Tan DT, Price FW, Jr. Endothelial keratoplasty: a revolution in evolution. Surv Ophthalmol. 2012;57(3):236-252.

2. Dapena I, Ham L, Melles GR. Endothelial keratoplasty: DSEK/DSAEK or DMEK--the thinner the better? Curr Opin Ophthalmol. 2009;20(4):299-307.

3. Dapena I, Moutsouris K, Ham L, Melles GR. Graft detachment rate. Ophthalmology. 2010;117(4):847-. e1.

4. Fernandez Lopez E, Baydoun L, Gerber-Hollbach N, Dapena I, Liarakos VS, Ham L, et al. Rebubbling techniques for graft detachment after Descemet membrane endothelial keratoplasty. Cornea. 2016;35(6):759-764.

5. O'Brien PD, Lake DB, Saw VP, Rostron CK, Dart JK, Allan BD. Endothelial keratoplasty: case selection in the learning curve. Cornea. 2008;27(10):1114-1148.

6. Parekh M, Ruzza A, Ferrari S, Ahmad S, Kaye S, Ponzin D, et al. Endothelium-in versus endothelium-out for Descemet membrane endothelial keratoplasty graft preparation and implantation. Acta Ophthalmol. 2017;95(2):194-198.

7. Romano V, Parekh M, Ruzza A, Willoughby CE, Ferrari S, Ponzin D, et al. Comparison of preservation and transportation protocols for preloaded Descemet membrane endothelial keratoplasty. Br J Ophthalmol. 2017. doi: 10.1136/bjophthalmol-2017-310906. 